\title{
LOCAL STARBURSTS IN A COSMOLOGICAL CONTEXT
}

\author{
Timothy M. Heckman \\ Center for Astrophysical Sciences \\ Johns Hopkins University \\ heckman@pha.jhu.edu
}

\begin{abstract}
In this contribution I introduce some of the major issues that motivate the conference, with an emphasis on how starbursts fit into the "big picture". I begin by defining starbursts in several different ways, and discuss the merits and limitations of these definitions. I will argue that the most physically useful definition of a starburst is its "intensity" (star formation rate per unit area). This is the most natural parameter to compare local starbursts with physically similar galaxies at high redshift, and indeed I will argue that local starbursts are unique laboratories to study the processes at work in the early universe. I will describe how NASA's GALEX mission has uncovered a rare population of close analogs to Lyman Break Galaxies in the local universe. I will then compare local starbursts to the Lyman-Break and sub-mm galaxies high redshift populations, and speculate that the multidimensional "manifold" of starbursts near and far can be understood largely in terms of the Schmidt/Kennicutt law and galaxy massmetallicity relation. I will briefly summarize the properties of starburst-driven galactic superwinds and their possible implications for the evolution of galaxies and the inter-galactic medium. These complex multiphase flows are best studied in nearby starbursts, where we can study the the hot X-ray gas that contains the bulk of the energy and newly produced metals.
\end{abstract}

Keywords:

\section{Introduction: What is a Starburst?}

Why are local $(z \ll 1)$ starbursts important? First of all, they are a very significant component of our present-day universe, and as such deserve to be understood in their own right. They provide roughly $10 \%$ of the radiant energy production and about $20 \%$ of all the high mass star formation in the local universe (e.g. Heckman 1998; Brinchmann et al. 2004). Their cosmological relevance has been highlighted by their many similarities to star forming galaxies at high-redshift. In particular, local UV-bright starbursts appear to be good analogs to the Lyman Break Galaxies (Meurer et al. 1997; Shapley et al. 2003; Heckman et al. 2004). Local starbursts provide a laboratory in which to 
study the complex ecosystem of stars, gas, black holes, galaxies, and the intergalactic medium up close and in detail. Finally, starbursts can contain millions of OB stars, and hence they also offer a unique opportunity to test theories of the evolution of massive stars.

Perhaps the most fundamental definition of a starburst would be that it is a galaxy in which the star formation rate approaches the upper limit set by causality. For a self-gravitating system this upper limit is reached if the entire gas reservoir is converted into stars in one dynamical time. For a total mass $M_{t o t}$, a gass-mass fraction $f_{\text {gas }}$, and a velocity dispersion $\sigma$, this upper bound can be written as

$S F R \leq M_{\text {tot }} f_{\text {gas }} / t_{\text {dyn }} \sim f_{\text {gas }} \sigma^{3} / G \sim 115\left(\sigma / 100 \mathrm{kms}^{-1}\right)^{3} f_{\text {gas }} M_{\odot} / y r$

If the star formation occurs with a standard IMF, the implied bolometric luminosity is:

$L_{\text {max }} \sim 10^{12}\left(\sigma / 100 \mathrm{kms}^{-1}\right)^{3} f_{\text {gas }} L_{\odot}$

Figure 1 compares this upper limit to observations of starburst galaxies in both the local universe and at high redshift. Starbursts approach the limit, and in extreme cases consistency requires gas-mass fractions approaching unity.

The classic definition of a starburst is in terms of its duration, and has two variations. First, a starburst is commonly defined as a galaxy in which the time it would take at the current star formation rate to consume the remaining reservoir of interstellar gas is much less than the age of the universe. This is commonly called the gas consumption time. The inverse of the gas consumption time is sometimes called the "efficiency". The related definition is that a starburst is a galaxy in which the time it would take to produce the current stellar mass at the current star formation rate is much less than a Hubble time. The inverse of this time can be recast as the "birthrate parameter" ( $b$, the ratio of the current to past average star formation rate).

These are sensible definitions and can be measured relatively easily. However, it important to note that the mass ratio of gas and stars varies significantly and systematically as a function of galaxy properties (e.g. Boselli et al. 2001). Thus (for example) using the birthrate parameter as the definition, leads to a steep decline in the fraction of starbursts with increasing galaxy mass (e.g. Brinchmann et al. 2004). On the other hand, since the gas mass fraction is so much smaller in massive galaxies, using the gas consumption time to define a starburst leads to very little mass-dependence of the starburst phenomenon. An additional point is that either of these timescale definitions of a starburst build in a strong redshift-dependence: since the age of the universe at $z \sim 5$ is only $10 \%$ of its present value, a galaxy with $b=10$ (strong starburst) today would have $b=1$ (not a starburst) at $z \sim 5$.

An alternative definition is that a starburst has a high intensity: the star formation rate per unit area $\left(I_{S F}\right)$ is very large compared to normal galaxies. As shown by Kennicutt (1998), this definition is functionally equivalent to the 


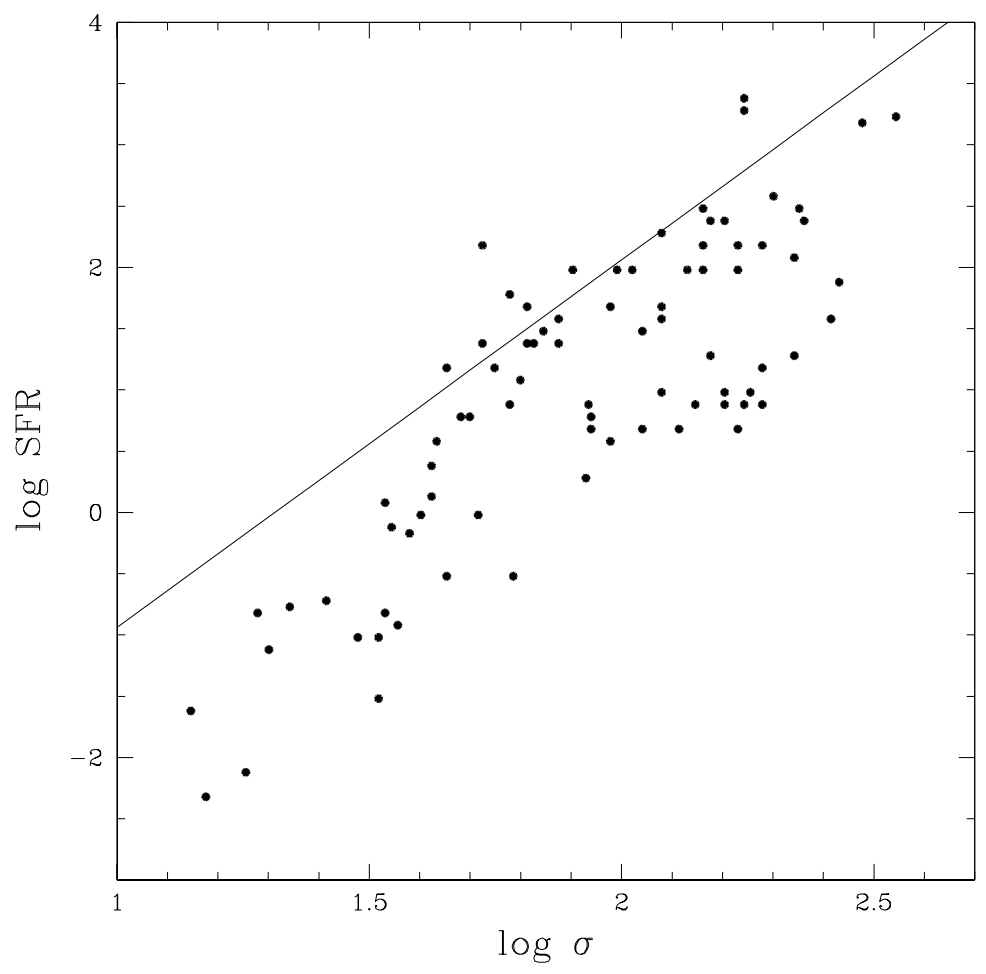

Figure 1. The log of the star formation rate $\left(M_{\odot}\right.$ per year) is plotted vs. the log of the galaxy velocity dispersion $(\mathrm{km} / \mathrm{sec})$ for the sample of local and high-redshift starbursts described by Murray et al. (2004). The diagonal line corresponds to the upper bound on the star formation rate allowed by causuality for a gas mass fraction of $100 \%$ (it corresponds to the conversion of the entire mass of the system into stars in a single dynamical time). See Heckman (1994) for an older version of this figure. Note that Murray et al. interpret this in terms of an upper limit on star formation set by the Eddington limit for radiation pressure acting on dust.

classic definition in terms of gas depletion time. Since he found that $I_{S F} \propto$ $N_{\text {gas }}^{3 / 2}$, this means gas depletion time $\propto I_{S F}^{-1 / 3}$. Extreme starbursts have star formation rates per unit area thousands of times larger than the disk of the Milky Way, and gas consumption times of only $\sim 10^{8}$ years. I will argue below that defining starbursts in terms of their intensity is the most physically useful way to proceed. 


\section{The Consequences of High Intensity}

The very high star formation rate per unit area in starbursts has immediate consequences for the basic physical properties of the galaxy. It immediately implies a high interstellar gas surface mass density $\left(\Sigma_{g}\right)$ (Kennicutt 1998) and also a high stellar surface mass density $\left(\Sigma_{*}\right)$. A typical case would have $I_{S F} \sim$ $10 \mathrm{M}_{\odot}$ year $^{-1} \mathrm{kpc}^{-2}$ and $\Sigma_{g} \sim \Sigma_{*} \sim 10^{9} \mathrm{M}_{\odot} \mathrm{kpc}^{-2}$. These are roughly $10^{3}$ $\left(\Sigma_{S F R}\right), 10^{2}\left(\Sigma_{g}\right)$ and $10^{1}\left(\Sigma_{*}\right)$ times larger than the corresponding values in the disks of normal galaxies.

The basic physical and dynamical properties of starbursts follow directly from the above. A gas surface-mass-density of $10^{9} \mathrm{M}_{\odot} \mathrm{kpc}^{-2}$ corresponds to an extinction of $A_{B} \sim 10^{2}$ for a Milky Way dust-to-gas ratio. The characteristic dynamical time in the star-forming region will be short: $t_{d y n} \sim(G \rho)^{-1 / 2} \sim$ $\left(G \Sigma_{t o t} H\right)^{-1 / 2} \sim$ few Myr, where $H \sim 10^{2} \mathrm{pc}$ is the thickness of the disk. A surface-brightness of a few $\times 10^{10} \mathrm{~L} \odot \mathrm{kpc}^{-2}$ corresponds to a radiant energy density inside the star-forming region that is roughly $10^{3}$ times the value in the ISM of the Milky Way. Finally, simple considerations of hydrostatic equilibrium imply correspondingly high total pressures in the ISM: $P \sim G \Sigma_{g} \Sigma_{t o t} \sim$ few $\times 10^{-9}$ dyne $\mathrm{cm}^{-2}\left(\mathrm{P} / \mathrm{k} \sim\right.$ few $\times 10^{7} \mathrm{~K} \mathrm{~cm}^{-3}$, or several thousand times the value in the local ISM in the Milky Way). The rate of mechanical energy deposition (supernova heating) per unit volume is also $10^{3}$ or $10^{4}$ times higher than in the ISM of our Galaxy.

As shown by Meurer et al. (1997), local starbursts and Lyman Break Galaxies have very similar values for $I_{S F}\left(1\right.$ to $100 M_{\odot} \mathrm{yr}^{-1} \mathrm{kpc}^{-2}$ ). Thus, this immediately implies that there are also strong similarities in the basic physical properties of local starbursts and Lyman Break Galaxies.

\section{Lyman Break Galaxy Analogs at Low Redshift}

While Meurer et al. (1997) showed that local starbursts and Lyman Break Galaxies have similar UV surface brightnesses, the former are generally smaller and less luminous than the latter. Local starbursts are either dwarf galaxies or small regions (usually nuclear) inside big galaxies. Are there true local analogs of the Lyman Break Galaxies in terms of size and ultraviolet luminosity? The success of NASA's Galaxy Evolution Explorer (GALEX - Martin et al. 2004) mission now makes it possible to find out.

We (Heckman et al. 2004) have used the first matched set of GALEX and Sloan Digital Sky Survey (SDSS) data to investigate the properties of a sample of 74 nearby $(z<0.3)$ galaxies with far-ultraviolet luminosities greater than $2 \times 10^{10} L_{\odot}$ (with no extimction correction). This was chosen to overlap the luminosity range of typical Lyman Break Galaxies. GALEX deep surveys have shown that ultraviolet-luminous galaxies similar to these are the fastest evolving component of the UV galaxy population (Arnouts et al. 2004; 
Schiminovich et al. 2004). Model fits to the combined GALEX and SDSS photometry yield typical FUV extinctions in these galaxies of 0.5 to 2 magnitudes (similar to Lyman Break Galaxies). The implied star formation rates are SFR $\sim 3$ to $30 M_{\odot} /$ year. This overlaps the range for Lyman Break Galaxies. We found a strong inverse correlation between galaxy mass and far-ultraviolet surface brightness, and on this basis divided our sample into "large" and "compact" systems. The large ultraviolet-luminous galaxies are relatively massive $\left(M_{*} \sim 10^{11} M_{\odot}\right)$ late type disk galaxies forming stars at a rate similar to their past average $\left(M_{*} / S F R \sim t_{\text {Hubble }}\right)$. They are metal rich $(\sim$ solar $)$, and have intermediate optical-UV colors ( $F U V-r \sim 2$ to 3$)$. In contrast, the compact ultraviolet-luminous galaxies have half-light radii of a few $\mathrm{kpc}$ or less (similar to Lyman Break Galaxies). They are relatively low-mass galaxies $\left(M_{*} \sim 10^{10} M_{\odot}\right)$ with typical velocity dispersions of 60 to $150 \mathrm{~km} / \mathrm{s}$. They span a range in metallicity from $\sim 0.3$ to 1 times solar, have blue optical-UV colors ( $F U V-r \sim 0.5$ to 2 ), and are forming stars at a rate sufficient to build the present galaxy in of-order a Gigayear. In all these respects they appear similar to the Lyman Break Galaxies.

The GALEX mission will ultimately find over a thousand such "living fossils" This will provide an opportunity for detailed local investigation of the physical processes occurring in typical star forming galaxies in the early universe.

\section{Understanding the Starburst Manifold}

Let us adopt the idea that a starburst by definition has a high star formation rate per unit area and a short gas depletion time. The most fundamental properties of a starburst would then be its star formation rate, metallicity, and dust opacity, and the mass of its host galaxy. In principle, starbursts could uniformly populate the multi-dimensional manifold defined by these parameters. Instead, them parameters show very strong systematic relations. More powerful local starbursts (higher SFR) are more metal rich, are more dust obscured, and occur in more massive galaxies (e.g. Heckman et al. 1998). At high redshift we know that the highest SFRs also occur in the most dust-obscured galaxies (the sub-mm sources compared to the Lyman Break Galaxies).

This systematic behavior can be understood in simple terms as the consequences of three effects. First, as discussed in the introduction above, causality implies that the maximum possible SFR is higher in more massive galaxies because they have higher velocity dispersions. This effect is mitigated somewhat by the systematically lower gas mass fraction $\left(f_{\text {gas }}\right)$ in more massive galaxies. Second, more massive star forming galaxies have systematically higher metallicity (Tremonti et al. 2004). Assuming a constant dust/metals ratio this implies that more massive galaxies have higher dust/gas ratios in the ISM. 
Third, the Schmidt-Kennicutt law implies that a high SFR requires a high ISM column density.

The natural assumption is that the amount of extinction in a starburst will be strongly related to the dust column density in the ISM (gas column density times dust/gas ratio). These three effects then natually explain why the more powerful starbursts are the more dust obscured (Wang \& Heckman 1996; Martin et al. 2004b). A higher SFR requires both a higher gas column and a more massive galaxy with its associated higher dust/gas ratio.

The argument can be easily extended to explain why galaxies with a given SFR are less dust-obscured at high redshift than in the local universe (Adelberger \& Steidel 2000). Consider a galaxy with a specified mass (stars plus gas plus dark matter). The Schmidt-Kennicutt law implies that the specific star-formation rate is $S F R / M \propto f_{\text {gas }} N_{\text {gas }}^{1 / 2}$. As long as $f_{\text {gas }}$ is systematically higher at higher redshift, this then implies that a correspondingly smaller $N_{\text {gas }}$ is required at higher redshift to sustain the same $S F R / M$ (e.g. if $f_{\text {gas }}$ increases by three, $N_{\text {gas }}$ would decrease by nine). If the mass-metallicity relation is displaced to lower metallicity (dust/gas ratio) at higher redshift this would only reinforce the effect.

The arguments above imply that both rest-frame UV and far-IR data are required to get a complete picture of the population of star forming galaxies, both locally at at high redshift. A UV(FIR)-selected sample will preferentially sample the population with lower (higher) metallicity, SFR, and mass (Martin et al. 2004b; Buat et al. 2004). In light of the above, it is natural to speculate that the main physical difference between the population of star forming galaxies at high-z selected as Lyman Break Galaxies or sub-mm sources is the galaxy mass. The latter may well be the progenitors of giant elliptical galaxies.

\section{Starburst-Driven Galactic Winds}

By now, it is well-established that galactic-scale outflows of gas are a ubiquitous phenomenon in the most actively star- forming galaxies in the local universe (see Heckman 2002 for a recent review). These outflows are potentially very important in the evolution of galaxies and the intergalactic medium. For example, by selectively blowing metals out of shallow galactic potential wells, they may explain the tight relation between the mass and metallicity in galaxies (Larson 1974; Tremonti et al. 2004). This same process would have enriched and heated the intergalactic medium in metals at early times (e.g. Adelberger et al. 2003), and explain why the majority of metals in galaxy clusters are in the intracluster medium (e.g. Loewenstein 2004).

We know that galactic winds are ubiquitous in the population of Lyman Break Galaxies (e.g. Shapley et al. 2003), and have also been observed in sub-mm-selected galaxies (Smail et al. 2003). The big advantage in studying 
them in local starbursts is that we can investigate their physics in considerably more detail, and in so-doing better understand the form and magnitude of the mass, energy, and metals being carried out in the wind.

Observations of local starburst winds show that they are complex multiphase phenomena. The hot $\left(\sim 10^{7} \mathrm{~K}\right)$ gas traced by X-ray emission appears to arise in shocks in the wind fluid as it impacts cooler denser material in the galaxy halo (e.g. Strickland et al. 2004; Lehnert, Heckman, \& Weaver 1999). As this ambient material encounters the wind it is heated and accelerated, giving rise to regions of optical line emission and the blueshifted interstellar absorption lines that are characteristic of Lyman Break Galaxies (Shapley et al. 2003) and local starbursts (e.g. Heckman et al. 2000). Dust contained in these clouds is revealed as it reddens the background starlight (Heckman et al 2000) and scatters the starburst's UV radiation (Hoopes et al. 2004).

The picture emerging from these panchromatic investigations of local starburst winds is that the fate of the outflow depends strongly on the phase of the outflow and the mass of the galaxy. The hot gas (which contains most of the energy and metals) has nearly the same temperature, independent of the escape velocity from the galaxy blowing the wind (Martin 1999). This hot gas is thus more likely to escape from low mass galaxies (with their shallower potential wells). This could naturally account for the galaxy mass-metallicity relation. In contrast, the outflow velocity in the cooler gas traced by the interstellar absorption lines is lower in the much less powerful starbursts in dwarf galaxies (Martin 2004). This is most likely because the low power winds in the dwarfs have insufficient thrust to accelerate interstellar clouds up to the velocity of the hot wind.

The combination of insights like these from the local universe and systematic investigations of the redshift dependence of the outflow rate derived through rest-frame UV spectroscopy will prove quite powerful in terms of addressing the cosmological significance of starburst-driven winds.

\section{Conclusions}

- Starbursts are an important component of the local universe and worth understanding in their own right.

- The key astrophysical property of a starburst is its "intensity" (SFR/area). This property has far-reaching consequences for the physical and dynamical properties of the ISM.

- Local starbursts provide excellent laboratories for the study of the astrophysics of high-z starforming galaxies, which have very similar SFR/area.

- GALEX has begun to provide a large sample of low-z analogs to Lyman Break Galaxies. 
- The systematic properties of starbursts are largely a consequence of the Schmidt/Kennicutt law plus the mass-metallicity relation. This explains why more massive galaxies host more powerful, more metal-rich, and more highly obscured starbursts. It also explains why high-z starbursts are less obscured on-average for a given SFR than low-z starbursts.

- Only in local starbursts can the multi-phase physics of galactic winds be fully investigated. The hot metal-rich phase traced by X-rays is the key to understanding how the IGM was chemically enriched by outflows from low mass galaxies.

\section{References}

Adelberger, K., \& Steidel, C. 2000, ApJ, 544, 218

Adelberger, K., Steidel, C., Shapley, A., \& Pettini, M. 2003, ApJ, 584, 45

Arnouts, S., and the GALEX Science Team 2004, ApJ, in press

Boselli, A., Gavazzi, G., Donas, J., \& Scodeggio, M. 2001, AJ, 121, 753

Brinchmann, J., Charlot, S., White, S., Tremonti, C., Kauffmann, G., Heckman, T., \& Brinkmann, J. 2004, MNRAS, 351, 1151

Buat, V., and the GALEX Science Team 2004, ApJ, in press

Larson, R. 1974, MNRAS, 169, 229

Heckman, T. 1994, in "Mass-Transfer Induced Activity in Galaxies", Edited by I. Shlosman, Cambridge University Press, p. 234

Heckman, T. 1998, in "Origins", ASP Conference Series, Vol. 148, 1998, ed. C. Woodward, J. M. Shull, and H. Thronson, Jr., p. 127

Heckman, T., Robert, C., Leitherer, C., Garnett, D., \& van der Rydt, F. 1998, ApJ, 503, 646

Heckman, T. 2002, in "Extragalactic Gas at Low Redshift", ASP Conference Proceedings Vol. 254. Edited by J. Mulchaey and J. Stocke, p. 292

Heckman, T., Lehnert, M., Strickland, D., \& Armus, L. 2000, ApJS, 129, 493

Heckman, T., and the GALEX Science Team 2004, ApJ, in press

Hoopes, C., and the GALEX Science Team 2004, ApJ, in press

Lehnert, M., Heckman, T., \& Weaver, K. 1999, ApJ, 523, 575

Loewenstein, M. 2004, in "Origin and Evolution of the Elements", Cambridge University Press, ed. A. McWilliam and M. Rauch, p. 425

Martin, C.L. 1999, ApJ, 513, 156

Martin, C.L. 2004, astro-ph/0410247

Martin, C.D., and the GALEX Science Team 2004a, ApJ, in press

Martin, C.D., and the GALEX Science Team 2004b, ApJ, in press

Meurer, G., Heckman, T., Leitherer, C., Lowenthal, J., \& Lehnert, M. 1997, AJ, 114, 54

Murray, N., Quataert, E., \& Thompson, T. 2004, astro-ph/0406070

Kennicutt, R. 1998, ApJ, 498, 541

Schiminovich, D., and the GALEX Science Team 2004, ApJ, in press

Shapley, A., Steidel, C., Pettini, M., \& Adelberger, K. 2003, ApJ, 588, 65

Smail, I., Chapman, S., Ivison, R., Blain, A., Takata, T., Heckman, T., Dunlop, J., \& Sekiguchi, K. 2003, MNRAS, 342, 1185

Strickland, D., Heckman, T., Colbert, E., Hoopes, C., \& Weaver, K. 2004, ApJS, 151, 193

Tremonti, C., Heckman, T., Kauffmann, G., Brinchmann, J., Charlot, S., White, S., Seibert, M., Peng, E., Schlegel, D., Uomoto, A., Fukugita, M., \& Brinkmann, J. 2004, ApJ, 613, 898

Wang, B. \& Heckman, T. 1996, ApJ, 457, 645 


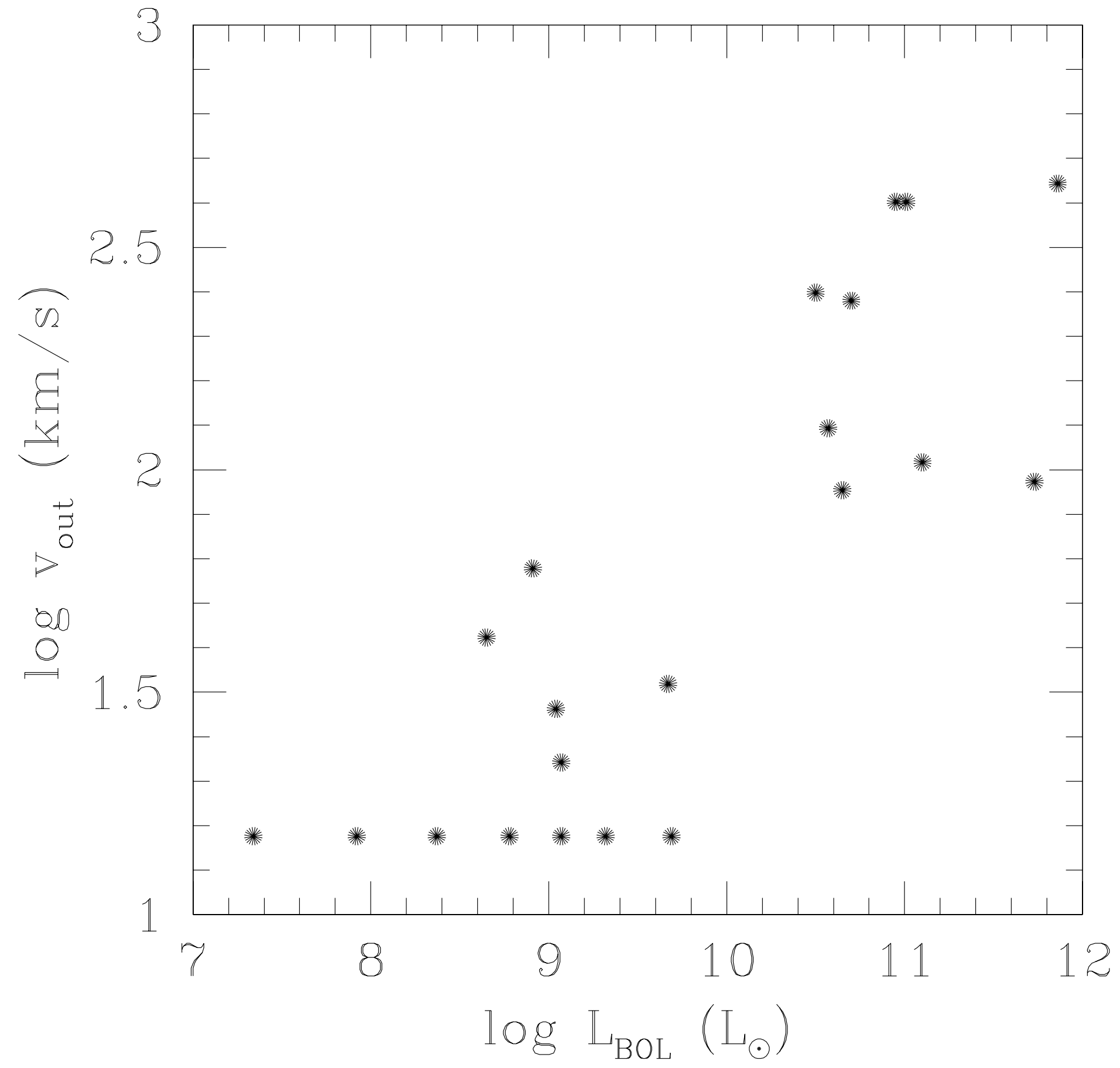

